\section{Preference for glucose disrupted by lateral preoptic lesions in the rat*}

\author{
DOUGLAS G. MOOK, GAYE P. LINDSEY, ELAINE K. PACE, \\ and R. CURTIS GRAEBER \\ University of Virginia, Charlottesville, Va. 22901
}

Rats with lateral preoptic lesions do not show the normal preference for dilute glucose solutions in brief-exposure situations. Some conditions for the occurrence of this deficit are identified.

Lesions in the lateral preoptic area (LPO) have been reported to produce a specific deficit in osmotic thirst in the rat (Blass \& Epstein, 1971). We have confirmed this finding and extended the investigation to the ingestion of fluids other than water.

Thirsty rats, when offered glucose solutions at various concentrations one at a time, display a characteristic intake pattern (cf. Epstein, 1967). As concentration rises over the low range, the amount drunk increases; with further increases in concentration, in t a k d e clines. This "preference-aversion function" is clearly visible in individual animals' data and is highly reliable. We have determined such functions for many rats in different contexts (Mook, 1963, 1969; Mook \& Blass, 1970; and the present control data), and every rat in our experience has shown such a pattern unless abnormalities were deliberately introduced.

In the present investigation, we determined preference-aversion functions in rats with verified deficits in osmotic thirst produced by LPO lesions. We found that, under appropriate conditions, rats with LPO damage show a greatly reduced preference or even a frank aversion to glucose solutions which intact rats, under comparable conditions, strongly prefer.

\section{METHODS}

Adult female albino rats (Sherman strain) were housed and tested in individual metal cages. Bilateral electrolytic lesions aimed at the LPO were placed at the following flat-skull coordinates: $0.7 \mathrm{~mm}$ anterior to bregma, $1.5 \mathrm{~mm}$ lateral from the sagittal sinus, and $7.3 \mathrm{~mm}$ ventral from dura. One milliampere dc was passed for 30 sec between the uninsulated tip of a stainless steel anode and a rectal cathode. Surgery was conducted under

*Supported by PHS Grant MH 10766. Address reprint requests to Douglas $G$. Mook, Department of Psychology, Gilmer Hall, University of Virginia, Charlottesville, Virginia 22901 .00 a $m$ following procedure are described below with results.

\section{RESULTS}

Four intact rats, when challenged with the hypertonic saline injection, all responded with vigorous drinking. They took in $10-14 \mathrm{ml}$ in the hour after injection and, in each case, somewhat more than half of the total intake occurred within the first 15 min. By contrast, in an initial series of five LPO rats, only three drank at all within the first $15 \mathrm{~min}$, and at the end of the hour, only those three had drunk as much as $2 \mathrm{ml}$. The data therefore support the finding (Blass \& Epstein, 1971) that LPO lesions can severely disrupt thirst induced by cellular dehydration. And since, as we shall see, the same rats drank normal amounts of water following fluid deprivation, even in the absence of food (cf. Kissileff \& Epstein, 1969), the data also support the conclusion that cellular dehydration is only one stimulus for drinking and that others are sufficient to support normal water intake.

In the experiments to follow, all LPO rats had verified osmotic-thirst deficits, in that they drank no more than $4 \mathrm{ml}$ in the hour following hypertonic saline injections.

Figure 1 shows single-bottle glucose preference-aversion functions in two LPO rats (the first two we observed). Look first at the filled circles, which standard procedure described above. In marked contrast to the rising and falling preference-aversion function which normal rats display (Fig. 2), the more dilute glucose solutions did not represent functions obtained under the \section{Modifications of this basic}

pentobarbital anesthesia $(145 \mathrm{mg} / \mathrm{kg}$ (0.16 mg ip).

After at least 1 week Glucose preference-aversio privation, the animals were of fere end of this maintenance period, water (but not food) was removed and the rat was deprived of fluid until the next

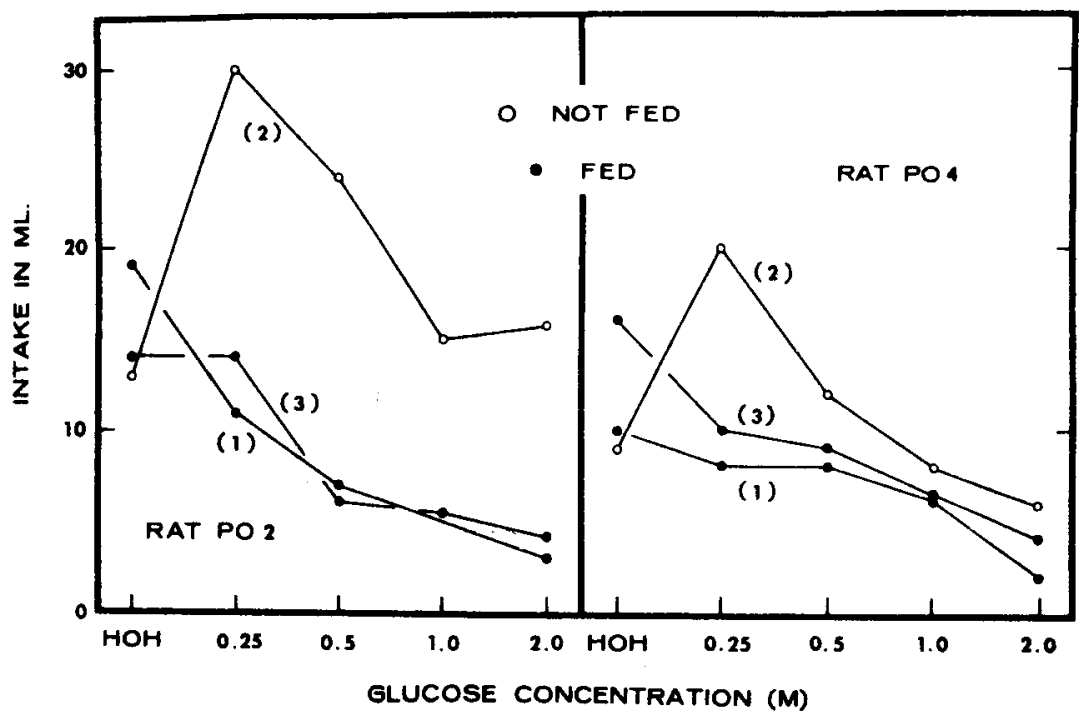

Fig. 1. Glucose preference-a version functions in two typical rats with LPO lesions. Measures were obtained following water deprivation, when food remained present ("fed") and when it did not ("not fed"). Numbers in parentheses represent the order in which the three functions were obtained. 


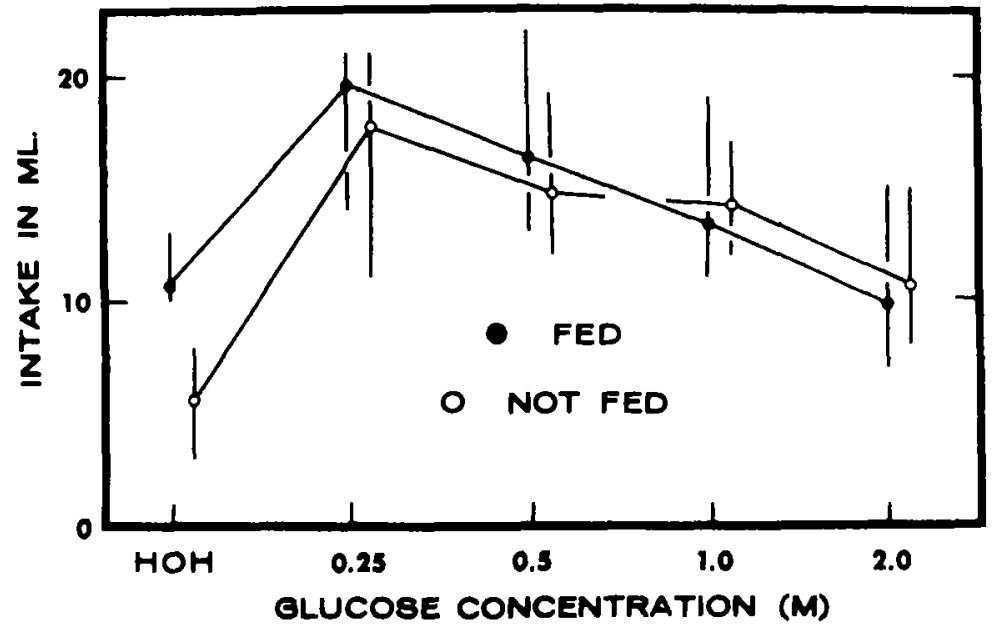

Fig. 2. Glucose preference-aversion functions in intact rats under "fed" and "not fed" conditions. The circles represent means, vertical lines represent ranges. The "not fed" data are displaced to the right for clarity.

elevate intake over the water baseline. Rather, addition of glucose to the drinking water depressed intake if it affected it at all. The single-bottle glucose preference is not expressed by such rats under these conditions.

These data were obtained following overnight deprivation of water, while food remained available (the "fed" condition). In the two rats shown in Fig. 1, we noticed that the animals seemed to have eaten rather large amounts of food overnight, in the absence of water. It occurred to us that perhaps, lacking the osmotic-thirst mechanism, these rats had a corresponding deficit in the normal suppression of food intake by dehydration (Adolph, 1947) and, therefore, were not hungry when the glucose solution was presented. Rats will treat sweet solutions either as fluid or as food (Teitelbaum \& Epstein, 1962; Collier \& Bolles, 1968), and we have elsewhere presented evidence (Knittle et al, 1970) that glucose drinking by water-deprived rats is driven primarily by hunger, not thirst. Perhaps, then, the rats we observed were eating the glucose, not drinking it; and perhaps our LPO rats, having eaten more food overnight, were less inclined to "eat" the glucose solution when it was offered.

We shall return to this idea, and we shall see that subsequent measurements make it hard to evaluate. But a preliminary test is straightforward. If LPO rats are made hungry as well as thirsty by withdrawal of both food and water overnight, the glucose preference should return. As the open circles ("not fed") in Fig. 1 show, this is the case: The normal strong glucose preference is reinstated under these conditions but again abolished by reimposition of the "fed" condition.
Thus, a major determinant of glucose preference in the LPO rat is the presence or absence of food during overnight water deprivation. This factor, as Fig. 2 shows, has negligible effects on glucose preference in intact rats, which eat little food in the absence of water.

Thirsty intact rats not only drink more of a dilute glucose solution than they do of water but they also select glucose in preference to water, if offered a choice between them (e.g., Young \& Greene, 1953; Mook, 1969). But the LPO rat does not. Six LPO rats and four controls were offered a choice between water and $0.25 \mathrm{M}$ glucose for 1 -h periods following overnight water deprivation ("fed") and food and water deprivation ("not fed"). The choice was offered twice under each condition, once with glucose to the left and once with water to the left.

As Table 1 shows, intact rats showed a strong glucose preference under both conditions; individual rats took $91 \%$ or more of their total intake from the glucose cylinder. The LPO rats also showed the glucose preference when deprived of both food and water. But when only water was withdrawn and food remained available prior to the test, such rats took in substantially less of their total intake $(8 \%-65 \%)$ as glucose $(p=.005)$. Only one LPO rat drank more glucose

Table 1

Mean Intake of Water and of $0.25 \mathrm{M}$ Glucose in Intact and LPO Rats After Deprivation of Water ("Fed") and Deprivation of Both Water and Food ("Not Fed")

\begin{tabular}{lrcccc}
\hline & \multicolumn{4}{c}{ Intake in Milliliters } \\
\cline { 3 - 5 } \multicolumn{1}{c}{ Ss } & Fed & & & Not Fed \\
\cline { 3 - 5 } & HOH & Glucose & & HOH & Glucose \\
LPO & 13.1 & 10.0 & 24.2 & 1.2 & 13.4 \\
\hline
\end{tabular}

than water, and some of these rats actively avoided it. Total fluid intake was about the same in both groups. Thus, under these conditions, the depression in glucose preference can reflect an actual aversion to a glucose solution which, in intact rats, is strongly preferred.

To summarize thus far, we know that LPO lesions can produce a deficit in the glucose preference, that the deficit may amount to a frank aversion to glucose, and that it has something to do with the presence of food during overnight water deprivation. Is water deprivation important? To answer this question, we offered the rats shown in Table 1 a choice between $0.25 \mathrm{M}$ glucose and water for $48 \mathrm{~h}$, with food available ad lib. The LPO rats, which had rejected this solution under the "fed" brief-exposure condition, all accepted it now, drinking on the average $76 \%$ of their total intake from the glucose cylinder (the average for intact rats was $87 \%$ ). Therefore, the severe preference deficit observed in the brief-exposure case does require that the animal be thirsty; a nonthirsty LPO rat, even if fed ad lib, does not display such a deficit.

Two conditions, then, are necessary to reveal the glucose preference deficit in the LPO rat. It must be deprived of water, and it must not be deprived of food. These are restrictive conditions, but the important point is that they produce no such preference disturbance in the intact rat.

Why should the deficit be specific to these particular conditions? As mentioned earlier, our first thought was that the LPO rat may reject glucose under such circumstances because it is not hungry. This in turn could result from its high intake of food, over the previous night, in the absence of water.

In the experiments summarized in Table 1, we measured overnight food intake during water deprivation, and it is clear that LPO rats do eat more than normals do. In the operated rats, food intake over the water-deprivation period ranged from 5.2 to $7.8 \mathrm{~g}$ per $100 \mathrm{~g}$ body weight; in intact rats, the corresponding values ranged from 3.1 to $4.9(p=.028)$. Further exploration, however, makes matters more complicated. In three LPO rats and three controls not used in previous 
Table 2

Average Overnight Food Intake (g/100 g Body Weight) in Intact and LPO Rats With and Without Water Available

\begin{tabular}{cccc}
\hline & Water & Percent \\
Ss & Present & $\begin{array}{c}\text { Water } \\
\text { Depression } \\
\text { in Absence } \\
\text { of Water }\end{array}$ \\
\hline LPO & 8.79 & Absent & 26 \\
Intact & 5.63 & 6.49 & 24 \\
\hline
\end{tabular}

experiments (but with verified osmotic-thirst deficits in the case of the operated rats), food intake during overnight water deprivation was compared with intake during the corresponding 16 -h period the preceding day, when water was available.

Table 2 shows the results. Food intake again is higher in LPO rats than in controls when water is withheld; but it also is higher even when water is available ( $p=.05$ in each case). And the proportion by which food intake is depressed in the absence of water is about the same in LPO rats as in intact ones.

Thus, while the deficit in glucose preference in LPO rats depends powerfully on the presence of food during overnight water deprivation, it is not clear whether aberrations in food consumption can account for it. Since such rats do eat more food than do normals during water deprivation, perhaps the absolute level of food intake is important. Or it may be that the consequences of feeding differ as between LPO rats and intact ones. Certainly the lesions do not disrupt a "preference mechanism" as such, for normal preference patterns are easily reinstated by a change in experimental condition.

Such brain damage does, however, reveal in magnified form the important role of hunger in the control of glucose intake. And it may provide a way of uncoupling hunger and thirst as determinants of the glucose preference.

\section{REFERENCES}

ADOLPH, E. F. Urges to eat and drink in rats. American Journal of Physiology, 1947, 151, 100-125.

BLASS, E. M., \& EPSTEIN, A. N. A lateral preoptic osmosensitive zone for thirst in the rat. Journal of Comparative \& Physiological Psychology, 1971, 76, 378-394.

COLLIER, G., \& BOLLES, R. Hunger, thirst, and their interaction as determinants of sucrose consumption. Journal of Comparative \& Physiological Psychology, 1968,66,633-641.

EPSTEIN, A. N. Oropharyngeal factors in feeding and drinking. In C. F. Code (Ed.) Handbook of physiology. Section 6: Alimentary canal, Vol.1. Washington, D.C: American Physiological Society, 1967.

KISSILEFF, $H . R$ \& EPSTEIN A. N Exaggerated prandial drinking in the "recovered lateral" rat without saliva. Journal of Comparative \& Physiological Psychology, 1969, 67, 301-308.

KNITTLE, E. A., LINDSEY, G. P. GRAEBER, R. C., \& MOOK, D. G. Depression of fluid preferences in rats with lateral preoptic lesions. Paper read at the Eastern Psychological Association meeting, Atlantic City, April 1970.

MOOK, D. G. Oral and postingestional determinants of the intake of various solutions in rats with esophageal fistulas. Journal of Comparative \& Physiological Psychology, 1963, 56, 645-659.

MOOK, D. G. Some determinants of preference and aversion in the rat. Annals of the New York Academy of Sciences, $1969,157,1158-1175$.

MOOK, D. G., \& BLASS, E. M. Specific hungers in hyperphagic rats. Psychonomic Science, $1970,19,34-35$.

TEITELBAUM, P., \& EPSTEIN, A. N. The lateral hypothalamic syndrome. Psychological Review, 1962, 69, 74-90.

WEINER, I. H., \& STELIAR, E. Salt preference of the rat determined by the single-stimulus method. Journal of Comparative \& Physiological Psychology, $1951,44,394-401$

YOUNG, P. T., \& GREENE, J. T. Quantity of food ingested as a measure of relative acceptability. Journal of Comparative \& Physiological Psychology, 1953, 46 288-294. 